\title{
A WIDE BAND MULTIPORT PLANAR POWER DIVIDER DESIGN BY RADIALLY COMBINING MATCHED SECTORIAL COMPONENTS
}

\author{
Yung-Jinn Chen and Ruey-Beei Wu \\ Department of Electrical Engineering, Rm. 340 \\ National Taiwan University. Taipei, Taiwan 10617, ROC
}

\begin{abstract}
This paper proposes a new multiport planar power divider design by radially combining the sectorial components and the input and output matching networks. It can achieve good input match over a wide bandwidth without resorting to transformer sections of high impedance lines which are difficult to realize. This approach is applied to design 4-way and 14-way center fed power dividers in microstrip structures with good input match $(V S W R<1.5)$ over a bandwidth of $30 \%$ and $15 \%$, respectively. A simple analysis method using the radial transmission line theory to model the microstrip sectorial components is presented to characterize the power divider. The calculated scattering parameters are found to be in good agreement with the measured data.
\end{abstract}

\section{INTRODUCTION}

Various designs of power dividers/combiners have been proposed in the literature, e.g., the survey paper [1]. One major problem associated with these power dividers is to achieve a good input match over a wide operation bandwidth. Theoretically, the dividers can include several transmission line sections of different impedances to provide good matching networks. The impedance levels will vary to a larger extent if either the number of output ports $\mathrm{N}$ or the desired bandwidth increases. In practice, it is hard to realize these impedance levels due to limitation of the achievable line width for microstrip or strip lines. For example, Hanczor and Kumar [2] proposed a 14-way power divider using maximally flat, two transformer sections. Their design called for two quarter wavelength sec-

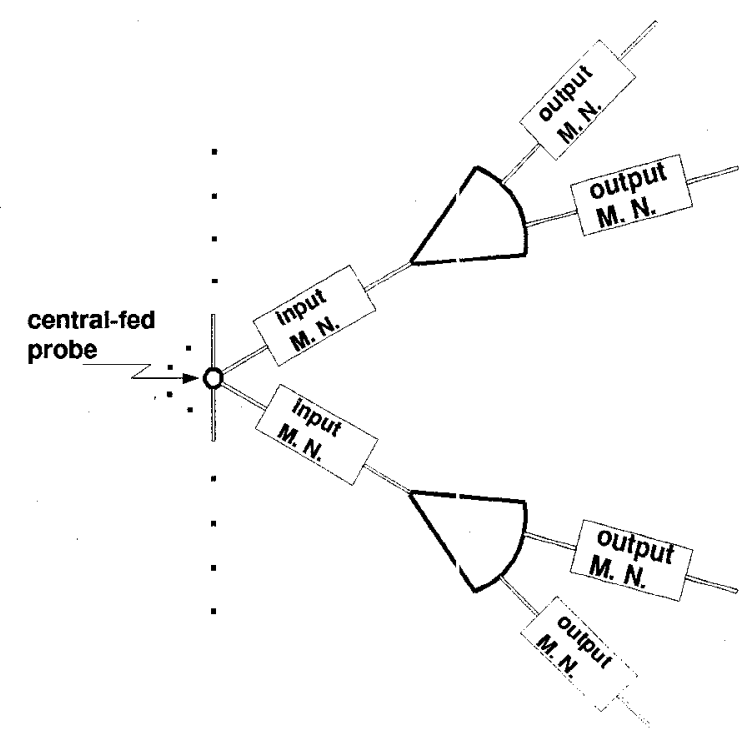

Fig.1: The whole topology of the proposed power divider design.

tions of characteristic impedances $37 \Omega$ and $140 \Omega$. The line of the characteristic impedance $140 \Omega$ is not adequate for microstrip nor strip lines unless some special substrate is used.

\section{DESCRIPTION FOR TOPOL- OGY}

Fig. 1 shows the topology of the $\mathrm{N}$-way power divider to be proposed in this paper. It consists of four parts: radial fan-out from the center-fed probe, input matching network, sectorial component, and output matching network. The sectorial 


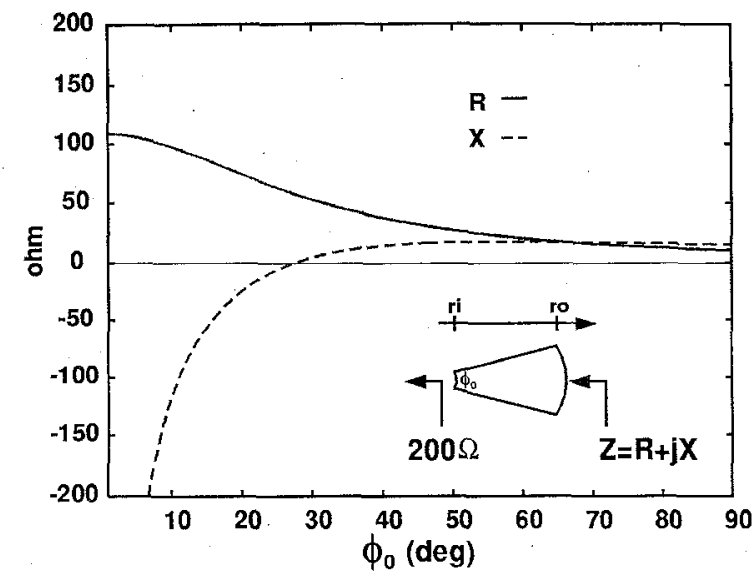

Fig.2: The impedance of the sectorial component of the 4-way power divider seen from the outer port versus the sector angle $\phi_{0}$ at frequency $f_{o}=3.3 \mathrm{GHz}$. The outer radius $r_{o}$ is $26.6 \mathrm{~mm}$.

component may connect one input port to one output port and serve as an impedance transformer. It may even connect one input port to two or more output ports and provide additional power dividing stage. Note that the topology may reserve its pure symmetry if the number of output ports connected to each sectorial component is no more than two. The matching network is made of three transmission line sections of different impedance levels and different lengths.

\section{EXPERIMENTAL AND THEO- RETICAL RESULTS}

Two examples are included to demonstrate the design procedure. The power dividers are all designed and measured on a microstrip structure with the substrate dielectric constant 4.33 and height $1.5 \mathrm{~mm}$. The output ports are $50 \Omega$ transmission lines which are realized by microstrip lines of width $2.89 \mathrm{~mm}$.

\section{A. 4-way power divider}

The first example is the design of 4-way power divider in the microstrip environment. The sectorial component works like a tapered transmission line, thereby achieving an impedance transform from the central probe to the output port. In the present case, consider the center frequency

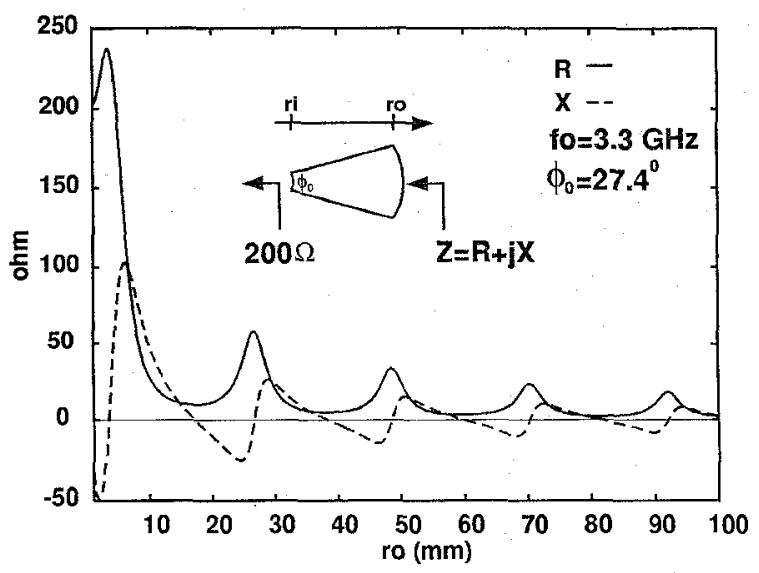

Fig.3: The impedance of the sectorial component of the 4-way power divider seen from the output port versus the outer radius $r_{o}$.

$f_{o}=3.3 \mathrm{GHz}$ and inner radius $r_{i}=1.0 \mathrm{~mm}$. From the requirement $J_{1}\left(k r_{o}\right) \cong 0$, the outer radius $r_{o}=26.6 \mathrm{~mm}$ is determined. By varying the sector angle $\phi_{0}$, Fig. 2 shows the impedance at the output port looking into the center probe which is of impedance $50 \cdot N=200 \Omega$. The imaginary part of the impedance is zero at $\phi_{0}=27.4^{0}$. The real part is about $58 \Omega$, a good impedance match to the output port. A thus designed power divider has been fabricated and measured. It shows good $V S W R$ at the center frequency, but of a rather narrow bandwidth. This occurrence can be accounted for Fig. 3 which exhibits a rapid impedance variation near the designed point of $r_{0}=26.6 \mathrm{~mm}$. For this sake, it is hard to achieve good impedance match by using sectorial component over a wide frequency band.

A design procedure to improve the wide band match is proposed. Based on the topology shown in Fig. 1, it starts with the selection of adequate radius and angle of the sectorial component. For example, $r_{o}$ is chosen to be $15 \mathrm{~mm}$ as evident from Fig. 3, where the impedance level of the sectorial component is comparatively flat. Although not shown here, the impedance curves versus $r_{o}$ with different sector angles $\phi_{0}$ have been investigated. It is found that a better impedance curve can be obtained by choosing a larger $\phi_{0}$, e.g., $\phi_{0}=51.4^{0}$. Given the geometric parameters, the generalized impedance matrix of the sectorial component can be extracted by radial transmission line theory [3]. Then, the impedance matrix is put into the 


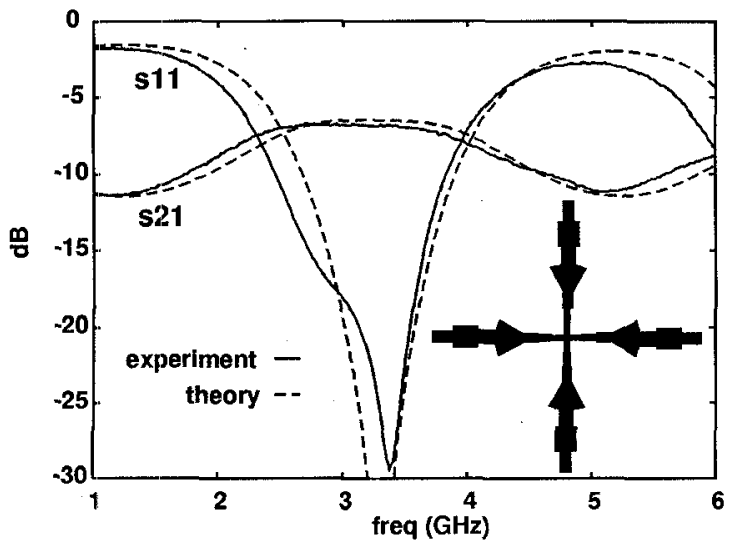

Fig.4: Calculated and measured $S_{11}$ and $S_{21}$ versus frequency for a 4 -way power divider design with both input and output matching networks. The geometrical parameters on input matching section (from probe to sector): $W_{1}=1.07, L_{1}=5.64$, $W_{2}=1.17, L_{2}=3.31, W_{3}=2.89, L_{3}=5$ $\mathrm{mm}$; on sectorial component: $\phi_{0}=51.4^{0}$, $r_{i}=3.33, r_{o}=15 \mathrm{~mm}$; on output matching section (from sector to output port): $W_{3}=4.96, L_{3}=5.94, W_{2}=6.97$, $L_{2}=5.89, W_{1}=2.89, L_{1}=10 \mathrm{~mm}$.

commercial software HP-EEsof to design the input and output matching networks for optimizing the bandwidth of the total topology.

Fig. 4 shows the scattering parameters versus frequency for the 4-way power divider with both input and output matching networks. It can be found that the theoretical and experimental results are in good agreement. The bandwidth of the power divider has been significantly enlarged by employing the present design procedure. Based on the criterion of $V S W R<1.5$, the bandwidth is as large as 30.8 percent.

\section{B. 14-way power divider}

The second example considers the design of a 14-way power divider, for which it is more difficult to achieve good input match due to a large $\mathrm{N}$, i.e., larger change of impedance level. According to the afore-mentioned design procedure, we select adequate outer radius $r_{o}=15 \mathrm{~mm}$ and sectorial angle $\phi_{0}=51.4^{0}$. The input and output matching sections are designed after an optimiza-

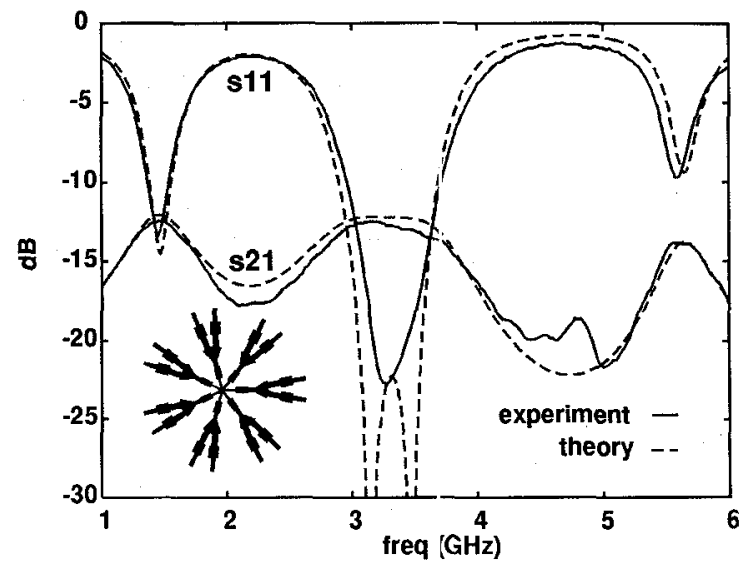

Fig.5: Calculated and measured $S_{11}$ and $S_{21}$ versus frequency for a 14 -way power divider design with both input and output matching networks. The geometrical parameters on input matching section (from probe to sector): $W_{1}=0.50, L_{1}=14.0$, $W_{2}=4.36, L_{2}=12.4, W_{3}=2.89, L_{3}=5$ $\mathrm{mm}$; on sectorial component: $\phi_{0}=51.4^{0}$, $r_{i}=3.33, r_{o}=15 \mathrm{~mm}$; on output matching section (from sestor to output port): $W_{3}=4.80, L_{3}=7.35, W_{2}=8.19$, $L_{2}=7.84, W_{1}=2.89, L_{1}=10 \mathrm{~mm}$.

tion by using HP-EEsof. The scattering parameters of this 14-way power divider design are shown in Fig. 5. High bandwidth and good input match are achieved in the frequency band of interest. In the figure, both the theoretical and experimental results are shown and found to be in good agreement. For the criterion $V S W R<1.5$, the divider exhibits good input match over a bandwidth of 15.7 percent centered at frequency $3.3 \mathrm{GHz}$.

For comparison sake, Hanczor and Kumar designed a 14-way power divider of similar performance [2]. Their design called for quarter-wave transformer sections with impedance level as large as $140 \Omega$. Such a high impedance line is quite difficult to realize in practical geometry if the substrate is present. The present design involves the matching transmission line sections of characteristic impedance at most $109 \Omega$ which is easier for realization by most transmission line configurations, e.g., microstrip lines. The highest impedance level of the transmission lines in the matching sections can be further reduced by tuning other lines in 


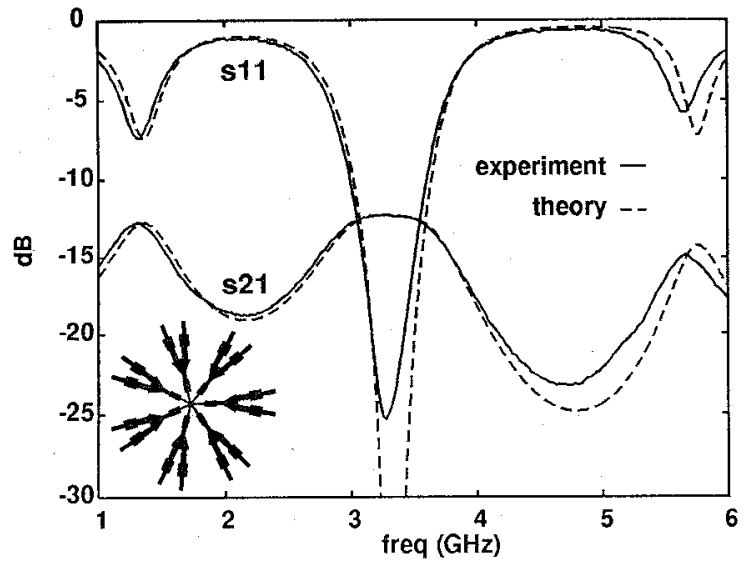

Fig.6: Another design for a 14-way power divider with both input and output matching networks. The geometrical parameters on input matching section (from probe to sector): $W_{1}=1.00, L_{1}=13.1$, $W_{2}=7.18, L_{2}=11.7, W_{3}=2.89, L_{3}=5$ mm; on sectorial component: $\phi_{0}=51.4^{0}$, $r_{i}=3.33, r_{o}=15 \mathrm{~mm}$; on output matching section (from sector to output port): $W_{3}=4.97, L_{3}=7.80, W_{2}=8.88$, $L_{2}=7.16, W_{1}=2.89, L_{1}=10 \mathrm{~mm}$.

the input and output matching networks. Shown in Fig. 6 are the scattering parameters of another design which has the highest impedance level of 85 $\Omega$. The design can still achieve good input match, but with a slight decrease in bandwidth as the tradeoff.

\section{CONCLUSIONS}

A new topology is proposed to design a wide band multiport planar power divider. The achievable bandwidth is $30 \%$ and $15 \%$ for the 4 -way and 14-way power dividers, respectively. Both the theoretical analysis and experimental measurement verify that the design based on the new topology can satisfy perfect balance of output powers and good input match over a wide bandwidth even when the number of output ports is large.

\section{References}

[1] K. J. Russell, "Microwave power combining techniques," IEEE Trans. Microwave Theory Tech., vol. MTT-27, pp. 472-478, May 1979.

[2] M. Hanczor and M. Kumar, "12-KW S-band solid-state transmitter for modern radar systems," IEEE Trans. Microwave Theory Tech., vol. MTT-41, pp. 2237-2242, Dec. 1993.

[3] A. Fathy and D. Kalokitis, "Analysis and design of a 30-way radial combiner for the $K u^{-}$ band applications," RCA Rev., vol. 47, pp. 487-508, Dec. 1986. 